\title{
La práctica docente mediada con tecnologías. YouTube como herramienta de aprendizaje en educación superior
}

\author{
Carmen Gallego Domínguez ${ }^{1}$ \\ Paulino Murillo Estepa²
}

Recibido 6 de noviembre 2018 · Aceptado 30 noviembre 2018

\begin{abstract}
RESUMEN
YouTube es uno de los portales de recursos audiovisuales más visitado en la red en el plano internacional. Se trata de una herramienta con un alto valor en el ámbito educativo, por su carácter motivador. Por ello, en este artículo se pretende conocer el potencial de uso de la herramienta cuando se utiliza en la práctica de aula en la educación superior. Este estudio responde a un diseño cuantitativo de corte descriptivo, en el que la muestra ha estado compuesta por ciento veinticuatro (I24) estudiantes de los diferentes grados impartidos en la Facultad de Ciencias de la Educación de la Universidad de Sevilla. Para recabar los datos se diseñó un cuestionario ad hoc, procediéndose a su análisis mediante el programa SPSS (v.2I), así como de la estadística descriptiva. Los resultados revelan tres aspectos de interés: a) YouTube, utilizado adecuadamente, es una herramienta útil y eficaz en el proceso de aprendizaje de los estudiantes; b) la creación de canales de contenidos educativos propios aporta importantes ventajas a la práctica docente en el aula, y c) es necesario el incremento de formación en competencias digitales en cuanto a la utilización de la herramienta como elemento innovador y motivador del aprendizaje.
\end{abstract}

Palabras clave: Competencia digital, herramienta didáctica, multimedia, educación superior, diseño de contenidos.

Teaching practice including technologies. YouTube as a learning tool in higher education

\section{ABSTRACT}

YouTube is one of the most visited audiovisual resource portals in the web. It is a high-value tool in education for it is highly motivating. This article aims to understand its potential in classroom practice in higher education and the study had a descriptive and quantitative design with a sample composed by one hundred twenty-four (I24) students of different grades taught in the School of Education Sciences at Universidad de Sevilla. In order to collect data, it applied an ad hoc questionnaire analyzed through the SPSS program (v.2I), as well as the descriptive statistics. Results show three interesting aspects: a) properly used,

I Doctora en Educación de la Universidad de Sevilla. Académica de la Universidad de Sevilla. cgallego@us.es

2 Doctor en Educación de la Universidad de Sevilla. Catedrático de la Universidad. paulino@us.es 
YouTube is a useful and effective tool in the learning process; b) creating channels of personal education content brings important advantages to the teaching practice in the classroom, and c) it is necessary to increase training in digital skills for using this tool as an innovative and motivating element for learning.

Keywords: Digital skills, didactic tool, multimedia, higher education, content design.

\section{Marco conceptual}

El desarrollo profesional docente comporta una formación basada en competencias que garantice que el profesorado integre saberes, funciones y tareas profesionales. Dicha integración resulta fundamental para la consecución de profesionales capaces en el desarrollo de procesos que conlleven y contemplen el conocimiento (saber), los procedimientos (saber bacer), los aspectos sociales (saber ser) y los participativos (saber estar), habilidades contempladas en el marco del Espacio Europeo de Educación Superior (EEES) como fundamentales para la formación docente, tanto desde el punto de vista profesional como disciplinar. En el mismo marco de referencia se reconoce la naturaleza innovadora que proporcionan las tecnologías cuando se integran en las prácticas docentes, contribuyendo igualmente al proceso de renovación impulsado por el EEES (Cañada Pujols, 2012; Mortis Lozoya, Valdés Cuervo, Angulo Armenta, García López \& Cuevas Salazar, 2013; Ricoy Lorenzo, Sevillano García \& Feliz Murias, 20I I; Ruiz Requies, Rubia Avi, Martínez Rodríguez \& Fernández Rodríguez, 2010). 
Figura I. Competencias del profesional docente según el Espacio Europeo de Educación Superior (EEES)

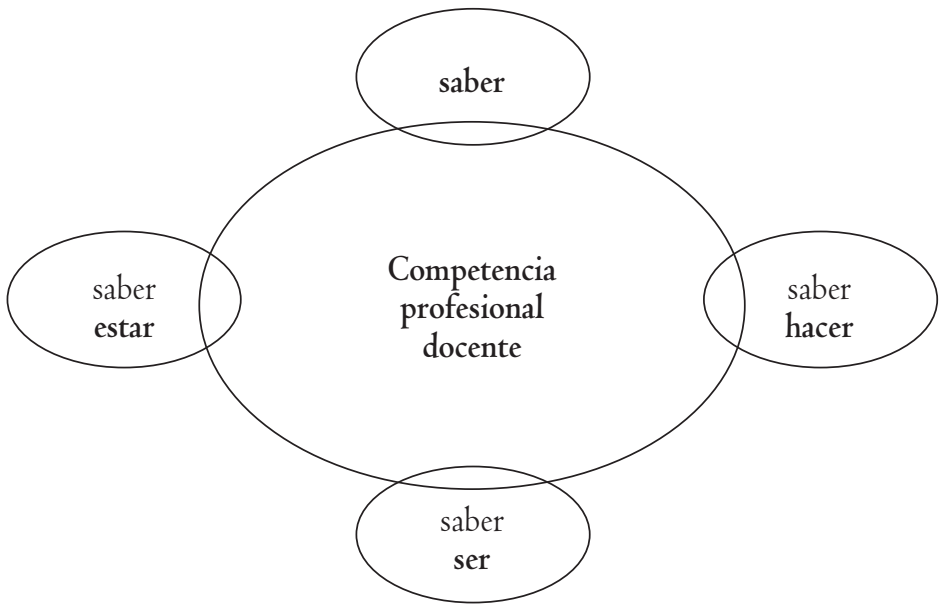

García-Valcárcel, Muñoz-Repiso y Tejedor Tejedor (2010, pp. I42 y I43) ofrecen un conjunto de competencias didácticas y pautas de mejora para integrar adecuadamente las TIC en la actividad docente desarrollada en las aulas. Espacios y tiempos, evaluación, actividades, materiales, comunidad educativa, organización del centro, formación y reconocimiento docente, y procesos de enseñanza-aprendizaje, constituyen las más relevantes.

Ricoy Lorenzo et al. (20I I), llaman la atención sobre la importancia de formar debidamente en competencias digitales a los estudiantes llamados a ser los docentes del mañana. Esto requiere la incorporación de modelos de formación orientados hacia el dominio de tales competencias. No se trata de usar tecnología, sino de integrarla en los procesos de enseñanza y de aprendizaje. Estos mismos autores delimitan el concepto de competencia como:

$[\ldots]$ la capacidad que se adquiere a través del aprendizaje y de la experiencia y que, combinando armónica y dinámicamente un conjunto de conocimientos, habilidades, destrezas, valores, actitudes y emociones, permite afrontar y resolver con éxito distintas situaciones o acciones contextualizadas por analogía o descubrimiento. (p. 485). 
De todos es sabido que Internet ofrece numerosas herramientas. En este sentido, Ricoy Lorenzo et al. (20II), destacan aquellas que un docente debe considerar para un desarrollo profesional competente, entre las que señalan las redes p2p, el e-mail, los foros formativos, el chat o Messenger, videoconferencia, edublogs, webquest, etc.

En la siguiente figura señalamos las competencias comunes a la tecnológica en general, independientemente de las herramientas concretas que utilicen los docentes.

Figura 2. Competencias comunes a la tecnológica general

Conocimientos y habilidades técnicas. Dominio técnico de programas y/o aparatos informáticos. Manejo de esta herramienta.

Juicio crítico. Enjuiciameitno crítico

Organización y planificación. Capacidad para organizar el contenido. Organizar y planificar la información.

Comunicación y lenguajes. Competencias comunicativas y de lenguajes de programación.

Búsqueda de información.

En definitiva, la utilización de herramientas TIC requiere el dominio de habilidades básicas y de conocimientos en el campo de la informática.

La cadena formativa que propone Monereo Font (2010), se concreta en las seis fases o eslabones que se detallan en la tabla I: la extrapolación, la simulación, la co-apropiación, la extensión, la participación restringida y la co-evolución. 
Tabla I. Eslabones de la cadena formativa (Monereo Font, 2010, p. 59I)

\begin{tabular}{|c|c|c|}
\hline FASE & DEFINICIÓN & EJEMPLO \\
\hline $\begin{array}{l}\text { La } \\
\text { extrapolación }\end{array}$ & $\begin{array}{l}\text { Conjunto de actividades } \\
\text { consistente en aplicar } \\
\text { determinados conceptos } \\
\text { a situaciones y problemas } \\
\text { que se producen } \\
\text { habitualmente en las } \\
\text { aulas. }\end{array}$ & $\begin{array}{l}\text { Experiencias personales, } \\
\text { noticias de actualidad, etc., } \\
\text { pueden constituir recursos } \\
\text { idóneos para efectuar esas } \\
\text { conexiones. }\end{array}$ \\
\hline La simulación & $\begin{array}{l}\text { Reproducir algunos } \\
\text { escenarios y } \\
\text { prácticas educativas } \\
\text { extraacadémicas, en el } \\
\text { aula universitaria. }\end{array}$ & $\begin{array}{l}\text { Especificación de las } \\
\text { decisiones de profesores } \\
\text { experimentados en la } \\
\text { planificación, desarrollo y } \\
\text { evaluación de una unidad } \\
\text { didáctica, con énfasis en las } \\
\text { situaciones inesperadas que } \\
\text { puedan aparecer y en el modo } \\
\text { de gestionarlas. }\end{array}$ \\
\hline $\begin{array}{l}\text { La } \\
\text { co-apropiación }\end{array}$ & $\begin{array}{l}\text { Resolución de problemas } \\
\text { profesionales en pareja. }\end{array}$ & $\begin{array}{l}\text { Cooperación entre ambos } \\
\text { para intentar solucionar el } \\
\text { problema en cuestión. }\end{array}$ \\
\hline La extensión & $\begin{array}{l}\text { Extender el aula } \\
\text { universitaria a las aulas } \\
\text { escolares en las que } \\
\text { trabajarán los futuros } \\
\text { docentes. }\end{array}$ & $\begin{array}{l}\text { Implicación directa de } \\
\text { profesores en ejercicio } \\
\text { para compartir con los } \\
\text { alumnos experiencias e } \\
\text { hitos profesionales, casos, } \\
\text { dificultades, concepciones } \\
\text { sobre su trabajo, etc. }\end{array}$ \\
\hline $\begin{array}{l}\text { La } \\
\text { participación } \\
\text { restringida }\end{array}$ & $\begin{array}{l}\text { Actividades a } \\
\text { desarrollar en el espacio } \\
\text { genuinamente profesional } \\
\text { del centro educativo. }\end{array}$ & $\begin{array}{l}\text { Estudiantes enfrentados a } \\
\text { tareas, problemas o actividades } \\
\text { profesionales reales, } \\
\text { previamente preparadas, con } \\
\text { el fin de minimizar posibles } \\
\text { errores y sus consecuencias. }\end{array}$ \\
\hline La co-evolución & $\begin{array}{l}\text { Realización de un } \\
\text { trabajo plenamente } \\
\text { profesional, junto con } \\
\text { otros, compartiendo los } \\
\text { diferentes procesos de } \\
\text { intervención de igual a } \\
\text { igual. }\end{array}$ & $\begin{array}{l}\text { Desarrollar un papel activo } \\
\text { en el que se recibe soporte } \\
\text { y formación, pero en el que } \\
\text { también se presta ayuda, } \\
\text { estableciendo una dinámica } \\
\text { de influencia bidireccional y } \\
\text { recíproca que pueda permitir } \\
\text { la evolución del equipo de } \\
\text { trabajo y la plena socialización } \\
\text { de todos sus miembros. }\end{array}$ \\
\hline
\end{tabular}


De todos modos, como desarrollo de la función docente, es imprescindible "conocer la auto-concepción de nivel de competencia digital propio con el que llegan los estudiantes. (...) esta información nos ayudará a generar unos procesos de formación y acreditación adecuados" (González Martínez, Espuny Vidal, de Cid Ibeas \& Gisbert Cervera, 2012, p. 29I).

Respecto de la formación inicial del docente para la integración de las TIC en su futuro profesional, tal y como afirman Ruiz Requies et al. (2010), hemos de considerar que modificar la práctica educativa no solo significa cambiarla como profesional, adquiriendo unas determinadas competencias. Significa, además, cambiarse a sí mismo, cambiar el contexto y el lugar de desempeño, poner en ejercicio la colaboración y el diálogo con los demás. Para que la integración de las TIC en los procesos formativos se desarrolle de forma innovadora es necesario que se produzcan cambios en el profesorado, en el alumnado, en las metodologías a desarrollar, así como en la propia institución.

Ruiz Requies et al. (2010), ofrecen una guía referida a las competencias en tecnología educativa, al tiempo que recuerdan que el aprendizaje por competencias no puede desarrollarse a través de la mera transmisión de conocimientos y contenidos, sino que la persona que se forma debe ser capaz de reflexionar, debatir, trabajar en grupo y aprender de forma activa y participativa. Igualmente, señalan las competencias generales y específicas, así como las tareas correspondientes a desarrollar (p. 165-169):

a) El aprendizaje a través de las TIC y el trabajo colaborativo.

b) La transmisión de información, ideas, problemas y soluciones a un público tanto especializado como no especializado.

c) La capacidad para analizar e incorporar de forma crítica el impacto social y educativo de los lenguajes audiovisuales y de las pantallas, así como las implicaciones educativas de las tecnologías de la información y la comunicación.

d) El diseño y la organización de actividades que fomentan en el alumnado los valores de no violencia, tolerancia, democracia, solidaridad y justicia, y reflexionar sobre su presencia en los contenidos de los libros de texto, materiales didácticos y 
educativos, y los programas audiovisuales en diferentes soportes tecnológicos destinados al alumnado.

e) Tareas relacionadas con la planificación y organización previa del trabajo.

f) Competencias de selección de contenidos, actividades, estrategias de enseñanza-aprendizaje y recursos tecnológicos.

g) Competencias en torno al proceso de evaluación.

h) Competencias interpersonales en torno a las TIC y al proceso colaborativo.

i) Competencias y destrezas tecnológicas.

j) Competencias identificadas por el alumnado relacionadas con la motivación en y hacia el proceso de enseñanza-aprendizaje.

Tierney (20I4), sostiene que los cambios formales tienen un verdadero y significativo impacto en la docencia. Del mismo modo, asegura que en la formación de los docentes juegan un papel importante los académicos o docentes con mayor experiencia, ya que guían y orientan a los que se están formando para que mejoren sus habilidades. No obstante, reconoce la resistencia al cambio por parte del sector docente, si bien parte de la consideración que la tecnología debe ayudar a la disolución de las mismas. El mismo autor señala ejemplos de periódicos, como el New York Times, que se vieron obligados a adaptarse a las tecnologías, porque en soporte papel empezaban a quedarse fuera del mercado.

Tierney, en sus planteamientos, introduce el término disruptive technology ${ }^{\mathrm{I}}$ para referirse a la necesaria adaptación del mundo educativo a las tecnologías con el fin de progresar y desarrollarse, así como para aumentar y mejorar el rendimiento de los estudiantes. Igualmente, señala que en la formación del profesorado novel interviene el proceso de mentoring ${ }^{2}$, es decir, la labor tutorial y orientativa de los docentes con experiencia en la enseñanza. Este aspecto es clave para que los

I "I have discussed the concept of disruptive technology to underscore the importance of adapting to societal change; new scholars need to become skilled with new technologies, develop innovative pedagogical approaches and write for multiple audiences and in multiple format" (Tierney, 2014, p. 697).

2 "Mentoring has proven to be successful under the right conditions insofar as the relationship between mentor and mentee can enable the early-career scholar to gain input and advice that is otherwise impossible" (Tierney, 2014, p. 692). 
docentes en formación puedan producir trabajos de calidad, ya no solo en su práctica diaria sino en sus investigaciones, tratando de sintetizar, aprender, leer, revisar y valorar sus trabajos académicos con ayuda de su supervisor o mentor de referencia.

Tierney (20I4), se pregunta: ¿por qué los cambios de la sociedad no pueden darse en la escuela? La teoría de la innovación disruptiva tiene explicación o poder en los retos y cambios a los que se enfrenta la educación. El aprendizaje en línea, o bien utilizar la tecnología como recurso educativo y de aprendizaje, es un proceso más cómodo, menos costoso y más fácil de utilizar, así como de personalizar. Es por ello que el profesorado debe formarse en habilidades y competencias en este campo, ya que los recursos e instrumentos a utilizar en el proceso de enseñanza-aprendizaje están cambiando y evolucionando constantemente.

En definitiva, la formación y la transformación desde las facultades, como instituciones de formación inicial de los docentes, deben contribuir al mejoramiento del desempeño futuro de la profesión, adaptándose a las necesidades sociales de una sociedad que se encuentra en constante cambio.

Los entornos de aprendizaje favorecedores para el aprendizaje del alumnado no dependen tanto del uso de las TIC en sí mismas, sino de la reorganización de la situación de aprendizaje y de la capacidad del profesorado para utilizar las TIC como soporte de transformación de las actividades de enseñanza que practica en su día a día en el aula (García-Valcárcel, Muñoz-Repiso \& Tejedor Tejedor, 2010).

Mortis Lozoya et al. (2013), señalan la importancia del uso de las TIC en la sociedad del conocimiento, así como el uso de las mismas por parte del alumnado para su adaptación a los constantes cambios a los que se tienen que enfrentar. Las TIC son esenciales para transitar de una sociedad informada a una sociedad más formada. Estos autores destacan tres competencias digitales necesarias para los docentes:

a) Instrumentales: conocimiento, uso funcional de los equipos y de los programas informáticos. Desarrollar procesos educativos, búsqueda, adquisición y procesamiento de la información. 
a. Utilizar los componentes básicos asociados a la tecnología: hardware, software, redes).

b. Utilizar software educativo libre.

c. Navegar en Internet.

d. Crear, modificar y refinar búsquedas en bases de datos electrónicas.

b) Cognitivas: reflexionar y aplicar criterios sobre el uso de las TIC en el aprendizaje y en la educación en general.

a. Interpretar normas éticas para el uso de las TIC en la práctica docente.

b. Conocer las normas e implicaciones legales del uso de las licencias para software comercial en la práctica docente.

c. Utilizar recursos digitales para detectar plagio en los trabajos escolares.

c) Didácticas-metodológicas: se requiere la integración de las TIC en los procesos de enseñanza-aprendizaje que tienen lugar en el aula. De esta forma se facilitará la creación y el diseño de unidades didácticas y actividades de aprendizaje.

a. Diseñar objetos de aprendizaje para usarlos en el fomento del aprendizaje.

b. Manejar actividades online que apoyen los procesos de aprendizaje en el estudiante.

Elaborar tutoriales a través de programas digitales.

YouTube, como herramienta, además de lo que nos pueda aportar como entretenimiento, puede convertirse en un interesante espacio educativo, en una herramienta con un enorme potencial de aprendizaje, de ahí que nos hayamos planteado su utilización como instrumento complementario en el desarrollo de nuestra práctica docente.

\section{Metodología}

El objetivo principal de esta experiencia, objeto del presente artículo, es conocer la predisposición de los estudiantes de grado de la Facultad de Ciencias de la Educación de la Universidad de Sevilla, para utilizar YouTube como una herramienta complementaria y de uso diario en la práctica docente. En este sentido, se concretan los siguientes objetivos 
específicos: (a) valorar la utilización de YouTube y los motivos para ello; (b) conocer la disposición a crear un canal propio de contenidos educativos; (d) conocer las necesidades formativas para incrementar el uso de YouTube en el aula.

Para el estudio, se ha utilizado una metodología cuantitativa y, en concreto, un diseño de corte descriptivo. Para seleccionar los estudiantes participantes se realizó un muestreo aleatorio simple, del que resultó un total de 124 estudiantes de los diferentes grados correspondientes a las titulaciones que se imparten en la Facultad. Se tomaron en consideración, en relación con los objetivos específicos planteados, las siguientes dimensiones para la construcción del instrumento de recogida de datos: (I) utilización de YouTube en el aula; (2) creación de un canal propio de contenidos, y (3) necesidades formativas en competencia digital.

Las dos primeras dimensiones se presentaron como preguntas abiertas, mientras que en la última se consideraron las aportaciones de la teoría revisada en cuanto a las áreas implicadas en el desarrollo de la competencia digital, construyéndose con ítems específicos respecto de las mismas. 
Tabla 2. Relación entre áreas de la competencia digital e ítems del instrumento

\begin{tabular}{|c|c|c|}
\hline ÁREA & COMPETENCIA DIGITAL & ÍTEM \\
\hline \multirow{3}{*}{ Información } & $\begin{array}{l}\text { Navegación, búsqueda y } \\
\text { filtrado de información }\end{array}$ & $\begin{array}{l}\text { Navegar, buscar y filtrar videos } \\
\text { para su uso en clase }\end{array}$ \\
\hline & Evaluación de información & $\begin{array}{l}\text { Evaluar videos adecuados para } \\
\text { los contenidos educativos }\end{array}$ \\
\hline & $\begin{array}{l}\text { Almacenamiento y } \\
\text { recuperación de la información }\end{array}$ & Almacenar y recuperar videos \\
\hline \multirow{2}{*}{ Comunicación } & $\begin{array}{l}\text { Interacción mediante nuevas } \\
\text { tecnologías }\end{array}$ & Interactuar con otros usuarios \\
\hline & $\begin{array}{l}\text { Compartir información y } \\
\text { contenidos }\end{array}$ & Compartir videos \\
\hline \multirow{3}{*}{$\begin{array}{l}\text { Creación de } \\
\text { contenido }\end{array}$} & Desarrollo de contenidos & $\begin{array}{l}\text { Crear y editar videos relacionados } \\
\text { con la temática a tratar }\end{array}$ \\
\hline & Integración y reelaboración & Subir videos \\
\hline & Derechos de autor y licencias & $\begin{array}{l}\text { Aplicar derechos de propiedad } \\
\text { intelectual y licencias de uso }\end{array}$ \\
\hline \multirow{4}{*}{ Seguridad } & \multirow{2}{*}{$\begin{array}{l}\text { Protección de datos personales } \\
\text { e identidad }\end{array}$} & Proteger datos personales \\
\hline & & Preservar la identidad \\
\hline & Protección de la salud & Proteger la salud visual \\
\hline & Protección del entorno & Proteger el entorno \\
\hline \multirow{2}{*}{$\begin{array}{l}\text { Resolución de } \\
\text { problemas }\end{array}$} & $\begin{array}{l}\text { Resolución de problemas } \\
\text { técnicos }\end{array}$ & Resolver problemas técnicos \\
\hline & $\begin{array}{l}\text { Innovación y uso de la } \\
\text { tecnología de forma creativa }\end{array}$ & Editar videos de manera creativa \\
\hline
\end{tabular}

Fuente: Elaboración propia

En cuanto al tratamiento de los datos, se ha utilizado el software de análisis estadístico SPSS (v.2I) y como técnica de análisis la estadística descriptiva para la escala que compone el cuestionario. Para los primeros apartados (cuestiones de carácter abierto) también se ha efectuado un análisis cuantitativo, partiendo de una codificación previa en función de las respuestas obtenidas.

\section{Resultados de la experiencia}

La sociología de la muestra participante en esta experiencia se distribuye con un 69,6\% de mujeres, frente al 30,4\% de hombres. 
Respecto de la edad, se diferencian tramos en grupos de 18 a 22 años (74,2\%), 23 a 28 años (24,2\%) y mayores de 29 años (I,6\%). En relación con la titulación se distingue el Grado en Educación Primaria con un 62,1\%, seguido de Infantil (13,7\%), Actividad Física y Deporte $(8,9 \%)$, Pedagogía (8,I\%) y otras titulaciones $(7,2 \%)$.

Finalmente, en referencia al curso al que pertenecen los estudiantes participantes en el estudio, los pertenecientes a segundo curso representan el 48,4\%, mientras que los de tercero suponen un $17,7 \%$, los de cuarto un I7\% y los de primero el 16,9\%.

\section{Utilización de YouTube en el aula}

Respecto del primer objetivo relacionado con la posibilidad de usar YouTube como una herramienta más en el desarrollo de las clases, un $92,7 \%$ responde de manera afirmativa, frente a un $7,3 \%$ que asegura que no la utilizaría.

Figura 3. Motivaciones que llevan a un uso de YouTube en clase.

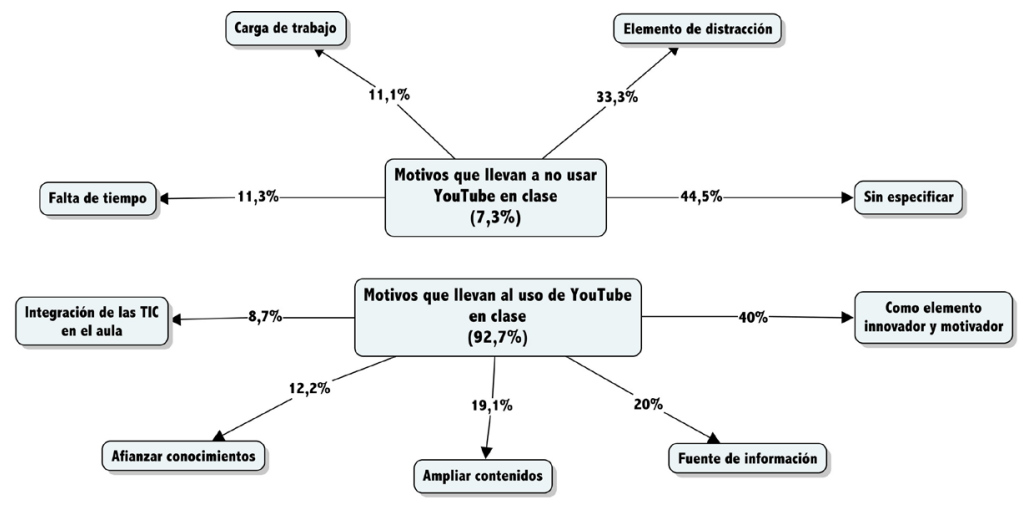

Fuente: Elaboración Propia, 2018

Entre los motivos que les llevarían a no utilizar YouTube en clase destacan la consideración de esta herramienta como un elemento de distracción (33,3\%), la carga extra de trabajo (I I,I\%) y la falta de tiempo (I I, I\%). Un 44,5\% no llega a especificar el motivo concreto de su no utilización. 
En cuanto a las razones de quienes manifiestan la idoneidad de utilización de la herramienta en clase, se señala la consideración de que supondría un método innovador, que contribuye a la motivación (40\%); mostrarse como una gran fuente de información (20\%); posibilita el poder ampliar contenidos mediante su uso (I9,I\%). Con porcentajes menores se señala la oportunidad para afianzar conocimientos (I2,2\%) o la posibilidad de integración de las TIC en el aula (8,7\%).

\section{Creación de un canal propio de contenidos}

En cuanto al segundo objetivo destinado a valorar si la muestra participante en esta investigación crearía un canal propio de YouTube para los contenidos educativos a trabajar, un 62,9\% afirma que sería un aspecto que tendrían en cuenta en su docencia mientras que un $37,1 \%$ responde de manera negativa.

Figura 4. Motivaciones que llevan a la creación de un canal educativo YouTube

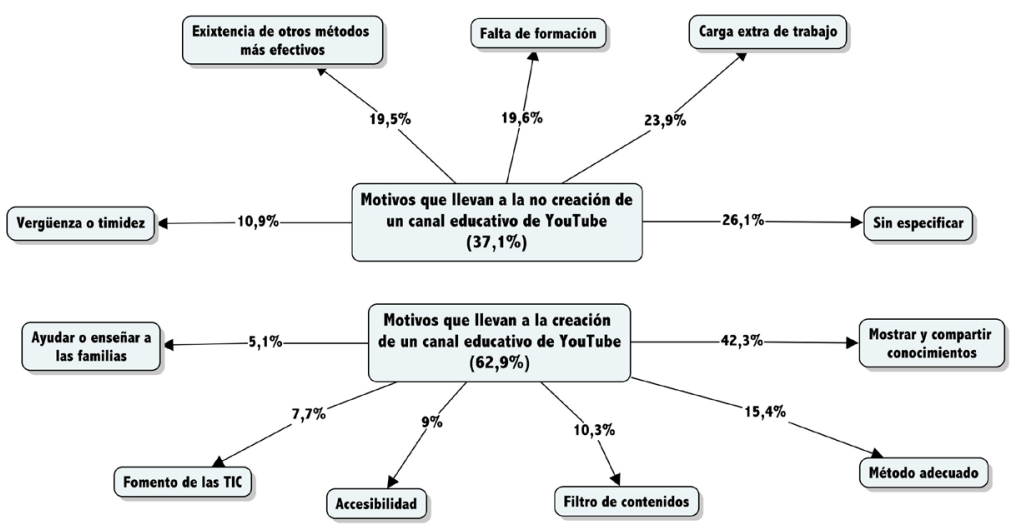

Fuente: Elaboración Propia

Entre los motivos por los que los estudiantes de titulaciones relacionadas con la educación no llevarían a cabo la creación de un canal propio de YouTube con contenidos educativos destacan la carga extra de trabajo que supondría $(23,9 \%)$, la falta de formación (I9,6\%) y la existencia de otros métodos más efectivos (I9,5\%). Por otro lado, conviene tener en consideración otros motivos diferentes 
señalados como la vergüenza o timidez (I0,9\%). Un 26,I\% no especifica su respuesta.

En relación con las razones por las que los estudiantes sí crearían un canal propio de contenidos educativos destaca la posibilidad de mostrar y compartir conocimientos (42,3\%), demostrar que se trata de un método adecuado y factible (I5,4\%), y la posibilidad de servir de filtro de contenidos adecuados, al ser realizado por personas con experiencia en el mundo educativo (I0,3\%). Otros aspectos se relacionan con la mejora de la accesibilidad ( $9 \%$ ), la posibilidad de repetición de los contenidos impartidos en clase, rompiendo barreras espaciotemporales (10,2\%), la integración de las TIC (7,7\%) y la posibilidad que brinda un canal de YouTube para contribuir a la colaboración de las familias (5,1\%).

\section{Necesidades formativas en competencia digital}

En cuanto al diagnóstico de necesidades formativas que harían aumentar el uso de YouTube como una herramienta más de clase, se ha tomado en consideración las aportaciones de las cinco áreas de competencia digital, obteniéndose los resultados porcentuales reflejados en el gráfico I, en función de las respuestas obtenidas en la escala de estimación del cuestionario. 
Gráfico I. Indicadores de las áreas de competencia digital relacionadas con el uso de YouTube.

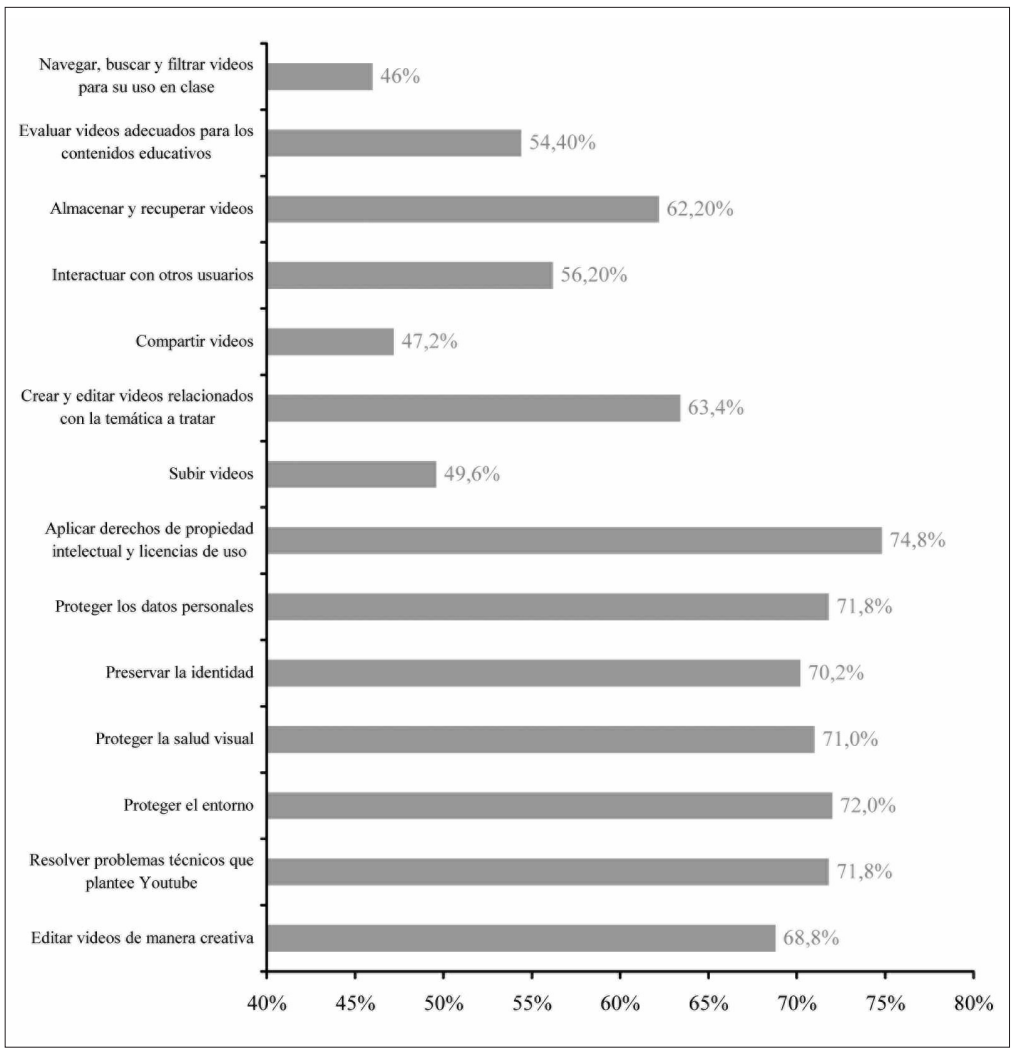

Fuente: Elaboración Propia, 2018

Por tanto, como se puede observar, en el gráfico I se presentan los resultados de la escala relacionada con las áreas de competencia digital vinculados a las necesidades formativas que, conforme se les fuera dando respuesta, supondría el incremento del uso de YouTube en las aulas como herramienta motivadora, así como la creación de canales educativos específicos.

En cuanto al área de "Información”, los ítems relacionados obtienen los siguientes porcentajes: Navegar, buscar y filtrar videos para su uso en clase un $46 \%$, Evaluar videos adecuados para los contenidos educativos un 54,4\% y Almacenar y recuperar videos un 62,2\%. Por lo tanto, en esta área de competencia digital los estudiantes participantes en esta experiencia 
muestran necesidades formativas de cierta importancia, lo que supone un dominio básico de los aspectos citados.

El área "Comunicación" se refleja en los ítems Interactuar con otros usuarios con un 56,2\% y Compartir videos con un $47,2 \%$. Se advierten, igualmente, necesidades de formación en dichos ámbitos, pero no tan altas como en otras áreas, por lo que YouTube mantendría su función como herramienta para el fomento de la interacción social, sin precisar de demasiada formación en los destinatarios de este estudio.

En relación con el área de "Creación de contenido" destacan Crear y editar videos relacionados con la temática a tratar con un $63,4 \%$, y Subir videos con un $49,6 \%$. En este sentido, esta área de competencia digital muestra la conveniencia de prestar atención a la creación de contenidos según la temática que se vaya a trabajar. La formación en este aspecto haría aumentar las posibilidades de creación de canales educativos.

Respecto del área "Seguridad" se muestran resultados más preocupantes. Ellos nos indican los siguientes porcentajes en cuanto a los ítems que tienen relación con la misma: Aplicar derechos de propiedad intelectual y licencias de uso (74,8\%), Proteger datos personales (71,8\%), Preservar la identidad (70,2\%), Proteger la salud visual (71\%) y Proteger el entorno (72\%). Esta área de competencia digital se presenta como prioritaria a la hora de atender a las necesidades formativas que se manifiestan, toda vez que una mayoría importante de estudiantes señala su falta de formación en las citadas competencias. Por lo tanto, todo hace indicar la necesidad de formación en cuanto a los elementos señalados, pues podría explicar los porcentajes de respuestas negativas obtenidos en relación con la creación de canales educativos de contenidos propios.

Finalmente, para el área "Resolución de problemas" desarrollada en los ítems Resolver problemas técnicos que plantee YouTube con un 7I,8\% y Editar videos de manera creativa con un $68,8 \%$, se evidencia también un alto grado de necesidades formativas. En este sentido, hay dos espacios en los que su formación conllevaría un aumento de creación de canales educativos, como serían la creatividad y la resolución de incidencias. 


\section{Conclusiones}

YouTube es considerada por los estudiantes participantes en esta experiencia como una herramienta educativa con un alto potencial de uso en la práctica diaria de clase, por sus múltiples ventajas metodológicas relacionadas con la innovación, aumento de la motivación, potenciación de ampliar y afianzar conocimientos e información, y la integración de las TIC en los procesos educativos.

No obstante, un pequeño porcentaje no mantiene esta concepción sobre YouTube, y sus motivos se basan, fundamentalmente, en aspectos como la falta de tiempo, la carga de trabajo o considerar que supone un elemento de distracción. Pensamos que esta opinión podría ser revertida con más formación en el conocimiento sobre las ventajas y posibilidades de esta herramienta, así como en programación y distribución del trabajo.

Además del mero uso de YouTube como una herramienta complementaria a la docencia, a la que se recurre en función de las necesidades de las sesiones, también se plantea un siguiente paso en la utilización de esta herramienta. Esto es, la creación de un canal propio en el que editar los contenidos a trabajar. En este sentido, la mayoría de los estudiantes concibe de manera positiva la edición de contenidos propios, debido a las ventajas que puede suponer, como poder mostrar y compartir conocimientos, usarla como una metodología adecuada, poner en marcha de un filtro de contenidos en los que el docente sería el evaluador de los materiales a disposición del alumnado, la mejora de la accesibilidad al conocimiento, la integración de las TIC en una sociedad cada vez más digitalizada y el aumento de la participación de las familias, gracias al acceso al conocimiento por medio del canal.

Por el contrario, existe una serie de motivos que los estudiantes argumentan para la no utilización de un canal de contenidos propios. Estos se basan en aspectos relacionados con la falta de formación en ámbitos académicos, organizativos y emocionales. Por lo que estos podrían ser fácilmente paliados con una formación específica adecuada. 
Sin embargo, los estudiantes que han participado en esta experiencia también muestran necesidades formativas relacionadas directamente con cada una de las cinco áreas de competencia digital, en lo referido a acciones que se relacionan con el uso de YouTube y la creación de canales educativos. Esta realidad muestra la necesidad de una más amplia formación digital a los estudiantes de titulaciones relacionadas con la educación, para así responder a una de las características que definen a la sociedad actual, como la predicción por medios tecnológicos.

Como elementos que han limitado esta experiencia, hemos de destacar la toma en consideración de una muestra de un contexto específico, sin tener la opción de compararla con otros escenarios. En este sentido, se ha tomado como muestra a estudiantes de titulaciones relacionadas con la educación. Por ello, esta experiencia podría ser replicada con docentes no universitarios en ejercicio, y así poder comparar los resultados que se obtengan, con el objeto de ver si en el desarrollo de la profesión se confirman las necesidades que el alumnado presenta en su época de estudiante.

\section{Referencias bibliográficas}

Cañada Pujols, M. D. (2012). Enfoque docente de la enseñanza y el aprendizaje de los profesores universitarios y usos educativos de las TIC. Revista de Educación, 359, 388-4I2. http://doi.org/I0.4438/I988-592XRE-20I I-359-099

García-Valcárcel Muñoz-Repiso, A. \& Tejedor Tejedor, F. J. (2010). Evaluación de procesos de innovación escolar basados en el uso de las TIC desarrollados en la Comunidad de Castilla y León. Revista de Educación, 352, I25-I47. Recuperado de http://www.revistaeducacion.mec.es/ re352/re352_06.pdf

González Martínez, J., Espuny Vidal, C., De Cid Ibeas, M. J. y Gisbert Cervera, M. (2012). INCOTIC-ESO. Cómo autoevaluar y diagnosticar la competencia digital en la Escuela 2.0. Revista de Investigación Educativa, 30(2), 287-302. http://doi.org/I0.6018/rie.30.2.II794I

Monereo Font, C. (2010). ¡Saquen el libro de texto! Resistencia, obstáculos y alternativas en la formación de los docentes para el cambio educativo. Revista de Educación, 352, 583-597. Recuperado de http://www. revistaeducacion.educacion.es/re352/re352_26.pdf 
Mortis Lozoya, S., Valdés Cuervo, A., Angulo Armenta, J., García López, R. I. \& Cuevas Salazar, O. (2013). Competencias digitales en docentes de educación secundaria. Municipio de un Estado del Noroeste de México. Perspectiva Educacional. Formación de Profesores, 52(2), I35-I53. http://doi. org/I0.4I5I/07I89729-Vol.52-Iss.2-Art.I74

Ricoy Lorenzo, M. C., Sevillano García, M. L. y Feliz Murias, T. (20II). Competencias necesarias para la utilización de las principales herramientas de Internet en la educación. Revista de Educación, 356, 483507. http://doi.org/I0-4438/I988-592X-RE-20I0-356-048

Ruiz Requies, I., Rubia Avi, B., Martínez Rodríguez, R. A. y Fernández Rodríguez, E. (2010). Formar al profesorado inicialmente en habilidades y competencias en TIC: perfiles de una experiencia colaborativa. Revista de Educación, 352, I49-I78. Recuperado de http://www.revistaeducacion. educacion.es/re352/re352_07.pdf

Tierney, W. G. (20I4). Academic competencies for the twenty-first century : plus ça change, plus c'est la même chose ? / Competencias académicas para el siglo XXI: plus ça change, plus c'est la même chose? Infancia $Y$ Aprendizaje: Journal for the Study of Education and Development, 37(4), 687-7I0. http://doi.org/I0.1080/02103702.2014.977109 